\title{
Conductores socioeconómicos sobre la lectura crítica según las pruebas Saber Pro en Colombia 2015
}

\author{
Eliana P. Londoño ${ }^{1}$, Vicky Ahumada ${ }^{2}$, Deyser Gutiérrez ${ }^{2}$ y María C. Gamboa ${ }^{2}$. \\ (1) Facultad de Ingeniería y Ciencias Ambientales. Fundación Universitaria Católica del Norte- FUCN. Medellín, \\ Colombia. (correo-e: eplondonog@ucn.edu.co) \\ (2) Escuela Ciencias de la Educación. Universidad Nacional Abierta y a Distancia UNAD. Grupo de Investigación \\ Ambientes de Enseñanza Aprendizaje de las Ciencias Básicas y Sociales - AMECI. Bogotá, Colombia. \\ (correo-e: vicky.ahumada@unad.edu.co, deyser.gutierrez@unad.edu.co,maria.gamboa@unad.edu.co) \\ * Autor a quien debe ser enviada la correspondencia
}

Recibido Nov. 6, 2019; Aceptado Ene. 6, 2020; Versión final Abr. 2, 2020, Publicado Ago. 2020

\section{Resumen}

El objetivo de este estudio es determinar la relación entre el rendimiento académico en las pruebas Saber Pro de 2015 en Administración de Empresas y en la Licenciatura en Etnoeducación. Se utilizaron 16 factores encontrados en las bases de datos del Instituto Colombiano de la Evaluación de la Calidad Educativa. Se utilizó como técnica estadística el ANOVA multivariado. Se encontró en la Licenciatura en Etnoeducación que factores como sector de la institución educativa superior, educación del padre, educación de la madre, ocupación de la madre y la modalidad de estudio inciden en el rendimiento. En Administración de Empresas los cuatro puntajes contribuyen al modelo mientras que en Etnoeducación son tres. En conclusión, en el modelo econométrico entre el rendimiento en lectura crítica y los puntajes de las pruebas Saber Pro comunicación escrita, inglés, razonamiento cuantitativo y competencias ciudadanas hay diferencias en el desempeño de los estudiantes por programa.

Palabras clave: competencias genéricas; evaluación; calidad educativa; pruebas estandarizadas; programas académicos presenciales y a distancia

\section{Socioeconomic conductors about critical reading according to Saber Pro tests in Colombia 2015}

\begin{abstract}
The aim of this study is to determine relationships between academic performance on the 2015 Saber Pro tests (Colombia) in the Business Administration program and the Ethno-education program. For this study, 16 factors were selected from the Colombian Institute of Evaluation of Educational Quality database and a multivariate ANOVA was employed for statistical analysis. The results from the Ethno-education program show that factors such as social sector of the higher educational institution, the father's education, the mother's education and job type, and the study mode have an impact on academic performance. In Business Administration and Ethno-education, there are four scores and three scores (respectively) that contribute to the model. In conclusion, in the econometric model there are differences on academic performance between critical reading and the test scores of the 2015 Saber Pro tests in the subject areas of written communication, English, quantitative reasoning, and citizen competence.
\end{abstract}




\section{INTRODUCCIÓN}

La calidad educativa o calidad en la construcción de conocimiento desde un marco epistemológico, se asocia al logro de las metas educativas relacionadas con el aprendizaje y el rendimiento académico independiente del contexto particular de los estudiantes, y a partir de las condiciones del proceso (Gómez et al., 2017). Por su parte la European Commission (2018) y Londoño (2013) señalan que la calidad no depende de un único aspecto, sino que está ligada a una serie de factores sociales, económicos, gubernamentales o personales de los actores académicos (docentes y estudiantes) y su impacto en el contexto donde se desenvuelven. Según la Organización de las Naciones Unidas para la Educación, la Ciencia y la Cultura (UNESCO), la calidad es un asunto relevante en la educación superior, asociado a la calidad de la enseñanza, de la formación y de la investigación; aspectos que se traducen en calidad del recurso humano y de la oferta académica (UNESCO, 1998). Otros aspectos a considerar son las interacciones entre los actores académicos, la dotación de infraestructura y de los entornos de la institución, y el acceso transparente a la información (De la Hoz, et al., 2019).

Buscando dar respuestas a las exigencias del mundo globalizado y la internacionalización de los saberes profesionales, el fomento del desarrollo y la calidad de la formación universitaria, el Ministerio de Educación Nacional (MEN) de Colombia identificó en 2008 cuatro competencias genéricas para la educación superior: 1) comunicación en lengua materna y otra lengua internacional, 2) pensamiento matemático, 3) ciudadanía y 4) ciencia, tecnología y manejo de la información. Así la calidad y la pertinencia de la Educación Superior dependen en gran medida de la promoción de estas competencias genéricas para la construcción de aprendizajes para toda la vida y la formación profesional con destrezas para un mundo cada vez más global; las tres primeras han sido evaluadas en las pruebas de educación superior en Colombia (MEN, 2009a; Bustamante et al., 2017).

La aplicación de pruebas de calidad de la educación superior se realizó en Colombia, por primera vez en 2003, bajo el nombre de Prueba ECAES (Exámenes de Calidad de la Educación Superior), fue reglamentada en el Decreto 1781 del mismo año; era una prueba oficial y de carácter obligatorio para los estudiantes próximos a graduarse y evaluaba competencias específicas y comunes para diferentes programas. Posteriormente, el Decreto 3963 de 2009, reglamenta esta prueba, como un requisito para obtener cualquier título profesional en Colombia; define tres objetivos principales: comprobar el grado de logro de las competencias en los estudiantes, utilizar la información como insumo para la comparación entre programas, instituciones y metodologías; y servir como fuente de información para elaborar indicadores de evaluación de la calidad, de manera que sea posible la cualificación de la institución, la formulación de políticas y la toma de decisiones (MEN, 2009b).

A partir del 2009 la prueba de Estado se denomina Saber Pro (Examen de Calidad de la Educación Superior). Al momento de inscribirse, el participante diligencia una encuesta de contexto que indaga por información: personal, académica y sociodemográfica. La prueba se realiza en dos sesiones, la primera conformada por cinco módulos que evalúan competencias genéricas: lectura crítica, razonamiento cuantitativo, competencias ciudadanas, comunicación escrita e inglés y la segunda entre uno y tres módulos que son específicos, de acuerdo con el área de formación del estudiante (ICFES, 2018). Estas últimas solo permiten comparaciones en el área de conocimiento respectivo y por ello no es posible comparar los logros entre programas, con las pruebas genéricas se supera esta situación, dado que permiten evaluar habilidades comunes independientes de la institución, el programa y la modalidad de estudio.

En Colombia la oferta académica de educación superior se realiza en dos modalidades: presencial y a distancia. La educación a distancia tiene dos metodologías: 1) distancia tradicional y 2) distancia virtual, con una creciente oferta de programas académicos de alta aceptabilidad (MEN, 2019). Bajo estas circunstancias, la evaluación y el control de la calidad se convierten, en aspectos necesarios, para mejorar el posicionamiento, y a partir de ello, las instituciones se han comprometido de manera más activa (Middlehurst, 2013). Una de las estrategias, tal vez la principal, en pro de lograr la calidad es la autoevaluación, abordando los aspectos pedagógicos y también los tecnológicos. Rausaria y Lele (2002), y Grifoll et al. (2010) recomiendan la autoevaluación, como práctica permanente en los programas académicos, clarificando las condiciones de calidad basadas en las particularidades metodológicas. Zhao et al. (2005) y Ahumada et al. (2019) señalan que existen estudios que demuestran que las propuestas formativas no tienen diferencia significativa en resultados, en cuanto a la modalidad, si se cumple la única condición de hacerlo con el rigor adecuado, garantizando el cumplimiento de los propósitos específicos de formación.

Un indicador de calidad de los programas académicos es la planeación consecuente con las necesidades, expectativas sociales y económicas, indistintamente de la modalidad. En este sentido, la Organización para la Cooperación y Desarrollo Económico (OCDE) citado en Gutiérrez et al. (2019) informa que Colombia entre el 2000 y el 2013, presentó un incremento del $48 \%$ en la matrícula de educación superior, sin embargo, está por debajo del incremento promedio de los países miembros, que es del $72 \%$; sin duda 
alguna, se hace necesario estudiar la pertinencia social de la oferta educativa y su calidad. Para Simonson et al. (2012) la educación a distancia y la presencial deben evaluarse a partir de criterios que midan la pertinencia con las necesidades de la sociedad y las necesidades laborales, para ello definen como fundamental el análisis de los materiales, la docencia y la creación de conocimiento. En la misma línea, Pham et al. (2018) se refieren a la pertinencia de la oferta formativa como un factor de calidad, la pertinencia vista como respuesta a las necesidades de la sociedad y a la oferta laboral, aspectos que están directamente relacionados con el origen y propósito de creación de los programas y sus currículos. Según Markova et al. (2017) la evaluación de la calidad de los programas se plantea a la luz de los procesos de interacción entre docentes y estudiantes, y los materiales de estudio que se ofrecen.

Damary et al. (2017) hacen referencia a la multiculturalidad de los estudiantes y lo complejo que resulta la evaluación de la calidad en distintos ambientes de aprendizaje, por cuanto, se presentan diferentes necesidades y expectativas, estos autores, hacen énfasis en la necesidad de ver la evaluación de la calidad educativa como una posibilidad de reflexión, autoevaluación y mejoramiento. Este estudio se centra en el tema de la calidad educativa dando una mirada al rendimiento académico de los estudiantes en pruebas Saber Pro, las competencias logradas y las variables que pueden estar influenciando sus resultados.

El rendimiento académico tiene factores que son necesario delimitar, entre éstos, vale la pena considerar el éxito-fracaso, medida con las calificaciones o los puntajes obtenidos en una prueba, al finalizar una experiencia de aprendizaje o luego de la aplicación de un examen estandarizado (Soria y Zúñiga, 2014). En este sentido para Edel (2003) y De Clercq et al. (2018) señalan que el rendimiento académico es multifactorial de orden cualitativo y cuantitativo, y considera dimensiones del perfil de un estudiante relacionado con sus conocimientos, habilidades, actitudes y valores logrados en un proceso de enseñanzaaprendizaje. Los factores o variables que influyen en el rendimiento académico pueden agruparse en: 1) individuales, correspondiendo a las características específicas del estudiante como estado fisiológico, inteligencia, motivación, estrategias de aprendizaje, entre otros, y 2) familiares, relacionados con el ingreso económico, posición social, nivel educativo de los padres, y socioculturales (Adeyemi y Adeyemi, 2014).

En cuanto a las condiciones individuales se ha encontrado que el apoyo social, entendido como la interacción familia-profesores-grupo de estudio y también las habilidades emocionales tienen efecto directo en el rendimiento académico (Olivos et al., 2016). En las familiares Edel (2003) incluye condiciones del entorno, recursos, niveles educativos de los padres y los eventos que pueden influir tanto positivamente o a la inversa en el desempeño académico al afectar la motivación, generar insatisfacciones o no encontrarle sentido a su esfuerzo; y en las socioculturales se relacionan las diferencias sociales, el entorno familiar, el nivel educativo del padre y la madre, el contexto socioeconómico y variables demográficas que influyen en los factores personales, y si el apoyo familiar es positivo y orientado al logro, específicamente si se asocian con la búsqueda de un proyecto de vida.

Con respecto a la articulación de la lectura crítica con el desarrollo de otras competencias, Martínez y González (2018), señalan que existe una relación al considerar que la lectura crítica es básica en el desempeño de cualquier profesional que se incorpora a la vida social, familiar, civil y productiva. En este sentido, la competencia de lectura crítica es de relevancia dado el efecto que tiene en la formación de profesionales que requieren el uso del lenguaje en su desempeño como un medio de expresión de comprensión de la realidad, hallando su lugar según las ideologías y las intenciones para objetar, refutar, cuestionar y argumentar; posibilita una formación profesional para una mejor interacción social (Betancourt y Frías, 2015).

\section{METODOLOGÍA}

El estudio corresponde a un enfoque cuantitativo y a un diseño ex post facto de tipo descriptivo y correlacional. Se empleó la base de datos del Instituto para la Evaluación de la Educación (ICFES) de Colombia, que contiene los resultados de pruebas estandarizadas que valoran competencias genéricas. Los análisis correspondientes se realizan a los resultados obtenidos por los estudiantes que aplicaron a la prueba Saber Pro en 2015 en los programas profesionales de Administración de Empresas y Licenciatura en Etnoeducación, encontrando 22.794 y 405 registros respectivamente.

El propósito del estudio es establecer si hay una relación estadísticamente significativa entre el rendimiento académico encontrado para estos programas profesionales con respecto a algunas de las variables socioeconómicas cuya respuestas se encontraron en la base de datos utilizada en este estudio, como también determinar si el rendimiento encontrado en la competencia de lectura crítica está influenciado por los resultados de las demás competencias genéricas como son comunicación escrita, inglés, razonamiento cuantitativo y competencias ciudadanas dado que cada una de las pruebas tiene un diseño independiente (ICFES, 2018). 
Las variables que se seleccionaron de la encuesta de contexto fueron: género del examinado, estado civil, carácter académico de la IES (Instituciones de Educación Superior), sector al que pertenece la IES, si estuvo becado, usó crédito para pagar los estudios, si los padres le pagaron la matrícula, el estudiante pagó sus estudios, si el estudiante es cabeza de familia, el nivel educativo del padre, de la madre, ocupación laboral del padre, de la madre, el estrato de su vivienda, si trabaja y la metodología de estudio. De las variables cuantitativas se seleccionaron los puntajes de las competencias genéricas como lectura crítica, comunicación escrita, inglés, razonamiento cuantitativo y competencias ciudadanas.

Los análisis estadísticos se realizaron con el paquete SPSS versión 19. Para determinar si el rendimiento académico obtenido en la prueba Saber Pro -que correspondió a la suma de los puntajes en cada una de las pruebas- está influenciado por las 16 variables de contexto, se empleó el análisis de varianza univariado para medir el efecto de cada una de las variables explicativas y las que se encontraron más fuertes se les realizó un ANOVA univariado múltiple. Y para determinar la relación del rendimiento en lectura crítica con los puntajes en las otras pruebas genéricas Saber Pro, se efectuó una regresión lineal múltiple usando como método de introducción de las variables el de selección hacia adelante que en cada paso genera un modelo conforme al grado de correlación que tenga con la variable dependiente. Se hicieron pruebas de colinealidad, cálculo del estadístico de Durbin-Watson, generación de gráficos de dispersión de los residuos controlando linealidad y homocedasticidad.

\section{RESULTADOS}

Para cada los programas de Administración de Empresas y la Licenciatura en Etnoeducación se presentan los estadísticos descriptivos del modelo econométrico del rendimiento académico en función de las variables socioeconómicas, los del resumen del modelo de ANOVA univariante multifactorial para el rendimiento académico. Luego se hace el análisis de la relación entre los puntajes entre lectura crítica y los de cuatro competencias genéricas restantes para estos programas. Acorde al modelo econométrico entre rendimiento académico logrado en pruebas Saber Pro 2015 y algunos factores socioeconómicos, los resultados encontrados para los dos programas: Administración de Empresas y Licenciatura en Etnoeducación fueron los siguientes: i) rendimiento académico en Saber Pro y las variables socioeconómicas para Administración de Empresas; ii) rendimiento académico en Saber Pro y los factores socioeconómicos para la Licenciatura en Etnoeducación; iii) análisis de la relación entre Lectura Crítica y cuatro competencias genéricas; iv) comportamiento en Saber Pro para Administración de Empresas; v) comportamiento en Saber Pro para la Licenciatura en Etnoeducación.

\section{Rendimiento académico y las variables socioeconómicas para Administración de Empresas}

Inicialmente, se valoró el rendimiento académico en las pruebas en función de cada una de las variables de contexto para determinar cuáles de ellas se relacionan mejor entre sí. La Tabla 1 contiene los resultados. En la Tabla 1 en todos los casos, los datos utilizados correspondieron a 22.794 registros, el alfa es igual a 0.05 . El test de Levene determina si las varianzas son homogéneas teniendo en cuenta el valor de $p$, en la tabla anterior se encontraron 13 factores que la cumplieron, mientras que en el test de efecto intersujetos indicando que todos no tienen diferencias en las medias intergrupales y la medida del efecto muestral señala que el factor no está explicando la mayoría de la variabilidad encontrada, mientras que al valorar el $\mathrm{R}^{2}$ ajustado se encontraron cuatro factores que sugieren alguna relación explicativa con el rendimiento académico, por lo que se realizó un ANOVA univariante multifactorial para determinar si es posible establecer un modelo. Los estadísticos se reportan en la Tabla 2. Se encontró un incremento en los valores de $R^{2}$ ajustados para cada uno de los efectos de los factores individuales aunque en la tabla se detectaron incrementos en los valores de $p$ en los efectos de dos de los factores y en nueve de las interacciones. El modelo solo explica el $4,6 \%$ de la varianza.

Tabla 1: Estadísticos descriptivos del modelo econométrico del rendimiento académico en función de las variables socioeconómicas en la carrera de Administración de empresas según la prueba Saber Pro 2015

\begin{tabular}{|l|l|l|l|l|l|l|}
\hline \multirow{2}{*}{ Variable explicativa } & \multicolumn{2}{l|}{ Test de Levene } & \multicolumn{2}{l|}{ Test del efecto intersujetos } & \multirow{2}{*}{$R^{2}$ ajustado } \\
\cline { 2 - 6 } & $F$ & $p$ & $F$ & $P$ & $\eta^{2}$ & \\
\hline Género & 139.506 & 0.000 & 7.044 & 0.008 & 0.000 & 0.000 \\
\hline Estado civil del examinado & 2.854 & 0.022 & 33.307 & 0.000 & 0.006 & 0.006 \\
\hline Carácter académico de IES* & 23.476 & 0.000 & 48.167 & 0.000 & 0.008 & 0.008 \\
\hline Sector al que pertenece IES* & 1.176 & 0.319 & 3.501 & 0.007 & 0.001 & 0.000 \\
\hline Fue becado & 0.386 & 0.680 & 19.978 & 0.000 & 0.002 & 0.002 \\
\hline Pagó la matricula a crédito & 6.192 & 0.002 & 11.233 & 0.000 & 0.001 & 0.001 \\
\hline Padres pagaron matrícula & 28.978 & 0.000 & 79.775 & 0.000 & 0.007 & 0.007 \\
\hline
\end{tabular}


Tabla 1: continuación

\begin{tabular}{|l|l|l|l|l|l|l|}
\hline \multirow{2}{*}{ Variable explicativa } & \multicolumn{2}{l|}{ Test de Levene } & \multicolumn{2}{l|}{ Test del efecto intersujetos } & \multirow{2}{*}{$R^{2}$ ajustado } \\
\cline { 2 - 6 } & $F$ & $p$ & $F$ & $P$ & $\eta^{2}$ & \\
\hline Estudiante pagó matrícula & 13.395 & 0.000 & 63.309 & 0.000 & 0.006 & 0.005 \\
\hline Estudiante es cabeza de familia & 0.121 & 0.886 & 65.640 & 0.000 & 0.006 & 0.006 \\
\hline Nivel educativo del padre & 12.223 & 0.000 & 29.668 & 0.000 & 0.020 & 0.020 \\
\hline Nivel educativo de la madre & 9.640 & 0.000 & 29.313 & 0.000 & 0.021 & 0.021 \\
\hline Ocupación laboral del padre & 11.456 & 0.000 & 9.414 & 0.000 & 0.007 & 0.006 \\
\hline Ocupación laboral de la madre & 7.443 & 0.000 & 10.298 & 0.000 & 0.008 & 0.007 \\
\hline Estrato donde vive & 32.001 & 0.000 & 70.103 & 0.000 & 0.018 & 0.018 \\
\hline El estudiante trabaja & 15.431 & 0.000 & 67.512 & 0.000 & 0.012 & 0.012 \\
\hline Modalidad de estudio & 7.232 & 0.001 & 47.860 & 0.000 & 0.004 & 0.004 \\
\hline
\end{tabular}

Tabla 2: Estadísticos resumen del modelo de ANOVA univariante multifactorial para el rendimiento académico en función de cuatro factores en Administración de Empresas para el 2015

\begin{tabular}{|c|c|c|c|c|c|c|c|}
\hline \multicolumn{8}{|c|}{ Prueba de igualdad de Levene de varianzas de error } \\
\hline \multicolumn{2}{|l|}{ Variable dependiente: } & \multicolumn{6}{|c|}{ Puntaje total obtenido por el estudiante } \\
\hline df1 & df1 & \multicolumn{3}{|l|}{$\mathrm{df} 2$} & \multicolumn{3}{|l|}{$p$} \\
\hline 1,484 & 1643 & \multicolumn{3}{|c|}{21150} & \multicolumn{3}{|l|}{0.000} \\
\hline \multicolumn{8}{|l|}{ Pruebas de efectos inter-sujetos } \\
\hline Variable dependiente: & \multicolumn{7}{|c|}{ Puntaje total obtenido por el estudiante } \\
\hline Origen & \multicolumn{2}{|c|}{$\begin{array}{l}\text { Tipo III de suma de } \\
\text { cuadrados }\end{array}$} & $g l$ & $\begin{array}{l}\text { Media } \\
\text { cuadrática }\end{array}$ & $F$ & $p$ & $\eta^{2}$ \\
\hline Modelo corregido & \multicolumn{2}{|l|}{$147568.528^{a}$} & 1643 & 89.817 & 1.673 & 0.000 & 0.115 \\
\hline Intersección & \multicolumn{2}{|l|}{597840.533} & 1 & 597840.533 & 11136.949 & 0.000 & 0.345 \\
\hline EDUCPADRE & \multicolumn{2}{|l|}{1487.806} & 10 & 148.781 & 2.772 & 0.002 & 0.001 \\
\hline EDUCMADRE & \multicolumn{2}{|l|}{1882.331} & 10 & 188.233 & 3.507 & 0.000 & 0.002 \\
\hline ESTRATO & \multicolumn{2}{|l|}{466.662} & 6 & 77.777 & 1.449 & 0.192 & 0.000 \\
\hline TRABAJA & \multicolumn{2}{|l|}{342.282} & 3 & 114.094 & 2.125 & 0.095 & 0.000 \\
\hline EDUCPADRE*EDUCMADRE & \multicolumn{2}{|l|}{4763.775} & 89 & 53.526 & 0.997 & 0.488 & 0.004 \\
\hline EDUCPADRE ${ }^{\star}$ ESTRATO & \multicolumn{2}{|l|}{3733.717} & 49 & 76.198 & 1.419 & 0.028 & 0.003 \\
\hline EDUCPADRE*TRABAJA & 1641.691 & & 27 & 60.803 & 1.133 & 0.289 & 0.001 \\
\hline EDUCMADRE*ESTRATO & 5550.590 & & 52 & 106.742 & 1.988 & 0.000 & 0.005 \\
\hline EDUCMADRE*TRABAJA & 1247.226 & & 30 & 41.574 & 0.774 & 0.805 & 0.001 \\
\hline ESTRATO*TRABAJA & 837.284 & & 16 & 52.330 & 0.975 & 0.481 & 0.001 \\
\hline EDUCPADRE`EDUCMADRE*ESTRATO & 16932.545 & & 331 & 51.156 & 0.953 & 0.721 & 0.015 \\
\hline EDUCPADRE*EDUCMADRE*TRABAJA & 13178.907 & & 228 & 57.802 & 1.077 & 0.204 & 0.011 \\
\hline EDUCPADRE*ESTRATO*TRABAJA & 6158.816 & & 127 & 48.495 & 0.903 & 0.774 & 0.005 \\
\hline EDUCMADRE*ESTRATO*TRABAJA & 6863.185 & & 125 & 54.905 & 1.023 & 0.413 & 0.006 \\
\hline $\begin{array}{l}\text { EDUCPADRE*EDUCMADRE ESTRATO* } \\
\text { TRABAJA }\end{array}$ & 24947.129 & & 497 & 50.195 & 0.935 & 0.845 & 0.022 \\
\hline Error & 1135349.335 & & 21150 & 53.681 & & & \\
\hline Total & 56851083.86 & & 22794 & & & & \\
\hline Total corregido & 1282917.863 & & 22793 & & & & \\
\hline a. $R^{2}=0,115\left(R_{\text {ajustada }}^{2}=0,046\right)$ & & & & & & & \\
\hline
\end{tabular}

\section{Rendimiento académico y los factores socioeconómicos para la Licenciatura en Etnoeducación}

Para este programa, de los nueve modelos obtenidos, el mejor modelo de regresión múltiple relacionó a la variable dependiente rendimiento académico en pruebas Saber Pro con los factores sector al que pertenece la IES, el estrato, el estado civil del estudiante y si el pagó su matrícula. Los diferentes resultados del modelo se describen en la Tabla 3. 
Tabla 3: Estadísticos descriptivos del modelo econométrico del rendimiento académico en función de los factores socioeconómicos en la carrera de Licenciatura en Etnoeducación según la prueba Saber Pro presentada en el año 2015

\begin{tabular}{|l|l|l|l|l|l|l|}
\hline \multirow{2}{*}{ Variable explicativa o factor } & \multicolumn{4}{l}{ Test de Levene } & \multicolumn{4}{l|}{ Test del Efecto intersujetos } & \multirow{2}{*}{$R^{2}$ ajustado } \\
\cline { 2 - 7 } & $F$ & $p$ & $F$ & $p$ & $\eta^{2}$ & \\
\hline Género & 14.416 & 0.000 & 1.010 & 0.315 & 0.003 & 0.000 \\
\hline Estado civil del examinado & 0.131 & 0.971 & 5.078 & 0.001 & 0.048 & 0.039 \\
\hline Carácter académico de IES & & & & & 0.000 & 0.000 \\
\hline Sector al que pertenece IES & 11.395 & 0.000 & 18.179 & 0.000 & 0.083 & 0.078 \\
\hline Fue becado & 1.928 & 0.147 & 2.987 & 0.052 & 0.015 & 0.010 \\
\hline Pagó la matricula a crédito & 0.183 & 0.833 & 3.069 & 0.048 & 0.015 & 0.010 \\
\hline Padres pagaron matrícula & 0.319 & 0.727 & 0.892 & 0.411 & 0.004 & -0.001 \\
\hline Estudiante pagón matrícula & 1.517 & 0.221 & 1.840 & 0.160 & 0.009 & 0.004 \\
\hline Estudiante es cabeza de familia & 0.809 & 0.446 & 1.516 & 0.221 & 0.007 & 0.003 \\
\hline Nivel educativo del padre & 1.514 & 0.132 & 1.921 & 0.041 & 0.046 & 0.022 \\
\hline Nivel educativo de la madre & 0.858 & 0.582 & 2.144 & 0.017 & 0.057 & 0.030 \\
\hline Ocupación laboral del padre & 0.607 & 0.837 & 0.940 & 0.507 & 0.028 & -0.002 \\
\hline Ocupación laboral de la madre & 0.487 & 0.922 & 1.485 & 0.127 & 0.043 & 0.014 \\
\hline Estrato donde vive & 0.401 & 0.879 & 8.584 & 0.000 & 0.115 & 0.101 \\
\hline El estudiante trabaja & 2.833 & 0.038 & 0.751 & 0.522 & 0.006 & -0.002 \\
\hline Modalidad de estudio & 1.634 & 0.196 & 23.813 & 0.000 & 0.106 & 0.101 \\
\hline
\end{tabular}

Según la Tabla 3 en todos los casos, los datos utilizados correspondieron a 405 registros, el alfa es igual a 0.05. Hay tres factores que cumplen con tener varianzas homogéneas según la prueba de Levene; en cuanto a las pruebas de efectos entre factores y rendimiento académico se encontraron ocho que son significativas al no tener diferencias en las medias intergrupales. Sin embargo, el valor del efecto muestra que diez de ellos podrían explicar las variaciones encontradas. Como en el caso de Administración de Empresas, se hizo la combinación de los factores que tuvieron un $\mathrm{R}^{2}$ ajustado igual o superior a 0,01 dejando a seis de ellos para la prueba de ANOVA univariada múltiple. Los resultados se encuentran en la Tabla 4. En el modelo se encontraron que cuatro factores no lograron significancia, lo mismo que 14 interacciones, también aparecieron 41 interacciones sin reporte de esos datos, aunque el valor del $\mathrm{R}^{2}$ ajustado indica que el modelo estaría explicando el $51,6 \%$ de la varianza.

Tabla 4: Estadísticos resumen del modelo de ANOVA univariante multifactorial para el rendimiento académico y seis factores en Licenciatura en Etnoeducación para el 2015

\begin{tabular}{|c|c|c|c|c|c|c|c|}
\hline \multicolumn{8}{|c|}{ Prueba de igualdad de Levene de varianzas de error } \\
\hline \multicolumn{2}{|c|}{ Variable dependiente: } & \multicolumn{6}{|c|}{ Puntaje total obtenido por el estudiante } \\
\hline $\mathrm{F}$ & df1 & \multicolumn{3}{|l|}{$\mathrm{df2}$} & \multicolumn{3}{|c|}{$p$} \\
\hline 3,288 & 271 & \multicolumn{2}{|l|}{133} & & \multicolumn{3}{|c|}{0,000} \\
\hline \multicolumn{8}{|c|}{ Pruebas de efectos inter-sujetos } \\
\hline \multicolumn{2}{|c|}{ Variable dependiente: } & \multicolumn{6}{|c|}{ Puntaje total obtenido por el estudiante } \\
\hline \multicolumn{2}{|l|}{ Origen } & $\begin{array}{l}\text { Tipo III de } \\
\text { suma de } \\
\text { cuadrados }\end{array}$ & $g l$ & $\begin{array}{l}\text { Media } \\
\text { cuadrática }\end{array}$ & $F$ & Sig. & $\eta^{2}$ \\
\hline \multicolumn{2}{|c|}{ Modelo corregido } & $17373.828^{a}$ & 271 & 64.110 & 2.589 & 0.000 & 0.841 \\
\hline \multicolumn{2}{|c|}{ Intersección } & 206922.118 & 1 & 206922.118 & 8355.249 & 0.000 & 0.984 \\
\hline \multicolumn{2}{|c|}{ SECTOR } & 120.688 & 2 & 60.344 & 2.437 & 0.091 & 0.035 \\
\hline \multicolumn{2}{|c|}{ EDUCPADRE } & 216.828 & 7 & 30.975 & 1.251 & 0.280 & 0.062 \\
\hline \multicolumn{2}{|c|}{ EDUCMADRE } & 108.580 & 9 & 12.064 & 0.487 & 0.881 & 0.032 \\
\hline \multicolumn{2}{|c|}{ OCUPMADRE } & 142.291 & 11 & 12.936 & 0.522 & 0.886 & 0.041 \\
\hline \multicolumn{2}{|c|}{ ESTRATO } & 27.130 & 6 & 4.522 & 0.183 & 0.981 & 0.008 \\
\hline \multicolumn{2}{|c|}{ MODEST } & 882.238 & 2 & 441.119 & 17.812 & 0.000 & 0.211 \\
\hline \multicolumn{2}{|c|}{ SECTOR ${ }^{\star}$ EDUCPADRE } & 140.206 & 3 & 46.735 & 1.887 & 0.135 & 0.041 \\
\hline \multicolumn{2}{|c|}{ SECTOR*EDUCMADRE } & 134.469 & 5 & 26.894 & 1.086 & 0.371 & 0.039 \\
\hline \multicolumn{2}{|c|}{ SECTOR*OCUPMADRE } & 116.616 & 1 & 116.616 & 4.709 & 0.032 & 0.034 \\
\hline \multicolumn{2}{|c|}{ SECTOR*ESTRATO } & 0.000 & 0 & & & & 0.000 \\
\hline \multicolumn{2}{|c|}{ SECTOR ${ }^{\star} M O D E S T$} & 11,329 & 1 & 11,329 & 0.457 & 0.500 & 0.003 \\
\hline \multicolumn{2}{|c|}{ EDUCPADRE*EDUCMADRE } & 491.270 & 13 & 37.790 & 1.526 & 0.116 & 0.130 \\
\hline \multicolumn{2}{|c|}{ EDUCPADRE*OCUPMADRE } & 816.877 & 10 & 81.688 & 3.298 & 0.001 & 0.199 \\
\hline \multicolumn{2}{|c|}{ EDUCPADRE*ESTRATO } & 17.866 & 5 & 3.573 & 0.144 & 0.981 & 0.005 \\
\hline \multicolumn{2}{|c|}{ EDUCPADRE*MODEST } & 2.700 & 1 & 2.700 & 0.109 & 0.742 & 0.001 \\
\hline \multicolumn{2}{|c|}{ EDUCMADRE*OCUPMADRE } & 379.597 & 15 & 25.306 & 1.022 & 0.437 & 0.103 \\
\hline \multicolumn{2}{|c|}{ EDUCMADRE*ESTRATO } & 46.473 & 6 & 7.745 & 0.313 & 0.929 & 0.014 \\
\hline \multicolumn{2}{|c|}{ EDUCMADRE*MODEST } & 17.557 & 3 & 5.852 & 0.236 & 0.871 & 0.005 \\
\hline OCUPN & & 13.198 & 3 & 4.399 & 0.178 & 0.911 & 0.004 \\
\hline
\end{tabular}


Tabla 4: continuación

\begin{tabular}{|c|c|c|c|c|c|c|}
\hline Origen & $\begin{array}{l}\text { Tipo III de suma } \\
\text { de cuadrados }\end{array}$ & $g l$ & $\begin{array}{l}\text { Media } \\
\text { cuadrática }\end{array}$ & $F$ & Sig. & $\eta^{2}$ \\
\hline OCUPMADRE*MODEST & 12.263 & 1 & 12.263 & 0.495 & 0.483 & 0.004 \\
\hline ESTRATO*MODEST & 1.118 & 2 & 0.559 & 0.023 & 0.978 & 0.000 \\
\hline EDUCPADRE*EDUCMADRE*OCUPMADRE & 301.882 & 3 & 100.627 & 4.063 & 0.008 & 0.084 \\
\hline EDUCPADRE*EDUCMADRE*ESTRATO & 1.834 & 1 & 1.834 & 0.074 & 0.786 & 0.001 \\
\hline OCUPMADRE*ESTRATO*MODEST & 47.065 & 1 & 47.065 & 1.900 & 0.170 & 0.014 \\
\hline Error & 3293.815 & 133 & 24.766 & & & \\
\hline Total & 934177.213 & 405 & & & & \\
\hline Total corregido & 20667.643 & 404 & & & & \\
\hline
\end{tabular}

\section{Análisis de la relación entre Lectura Crítica y cuatro competencias genéricas}

Otro propósito de este estudio es determinar cuál de los resultados en las pruebas individuales de comunicación escrita, inglés, razonamiento cuantitativo y competencias ciudadanas se relacionan con el puntaje obtenido en lectura crítica. Para la realización de esta prueba en el SPSS versión 19, se consideraron las siguientes hipótesis: (a) todas las variables independientes son determinantes ya que provienen de una muestra, (b) existe una variable error en el modelo que tiene una media de cero y su varianza no depende de los datos presentándose la homocedasticidad, (c) la variable dependiente es aleatoria y es afectada por la variable error, (d) todas las variables independientes son es relevante en la explicación del modelo y no hay errores de especificación, (e) todas las variables independientes o explicativas no tienen ninguna relación entre sí (no hay multicolinealidad) y (f) la variable de error tiene una distribución normal en todo el rango de variación de los datos.

\section{Comportamiento en Saber Pro para Administración de Empresas}

Al efectuar la regresión múltiple para este programa se encuentran como estadísticos descriptivos los reportados en la Tabla 5. En la Tabla 5 todos los casos, los datos utilizados correspondieron a 22.794 registros. Para valorar las condiciones de fiabilidad y validez del modelo de regresión múltiple entre lectura crítica y los resultados de las pruebas de comunicación escrita, inglés, razonamiento cuantitativo y de competencias ciudadanas, las Tablas 6 y 7 muestran los resultados encontrados.

Tabla 5: Estadísticos descriptivos de las competencias genéricas Saber Pro en la carrera de Administración de Empresas presentados en el año 2015

\begin{tabular}{|l|c|c|}
\hline Variable & Media & Desviación estándar \\
\hline Puntaje en lectura crítica & 10.083 & 1.6064 \\
\hline Puntaje en comunicación escrita & 9.691 & 1.7061 \\
\hline Puntaje en inglés & 9.989 & 1.8330 \\
\hline Puntaje en razonamiento cuantitativo & 9.804 & 1.6381 \\
\hline Puntaje en competencias ciudadanas & 9.807 & 1.5917 \\
\hline
\end{tabular}

Tabla 6: Estadísticos resumen del modelo de regresión lineal para lectura crítica

\begin{tabular}{|c|c|c|c|c|c|c|}
\hline Pruebas & \multicolumn{6}{|c|}{ Valores estadísticos } \\
\hline \multirow{3}{*}{$\begin{array}{l}\text { Regresión } \\
\text { múltiple }\end{array}$} & \multirow[t]{2}{*}{$\mathrm{R}^{2}$ ajustado } & \multirow[t]{2}{*}{ Error estándar } & \multicolumn{3}{|c|}{ Estadísticos de cambio } & \multirow[t]{2}{*}{ Durbin-Watson } \\
\hline & & & $\mathrm{R}^{2}$ ajustado & $\mathrm{F}$ & Sig. $F$ & \\
\hline & 0.840 & 0.6148 & 0.840 & 29995.962 & 0.000 & 1.995 \\
\hline \multirow[t]{4}{*}{ ANOVA } & Estimados & Suma cuadrados & gl & Media cuadrática & $\mathrm{F}$ & Sig. \\
\hline & Regresión & 49426.705 & 4 & 12356.676 & 29995.962 & 0.000 \\
\hline & Residuo & 9387.807 & 22789 & 0.412 & & \\
\hline & Total & 58814.511 & 22793 & & & \\
\hline
\end{tabular}

Tabla 7: Coeficientes para el modelo de regresión múltiple de lectura crítica en Administración de Empresas

\begin{tabular}{|l|l|l|l|l|l|}
\hline Variable & $\begin{array}{l}\text { Coeficientes } \\
\text { estandarizados }\end{array}$ & $t$ & \multirow{2}{*}{ Sig. } & \multicolumn{2}{|l|}{ Estadísticas colinealidad } \\
\cline { 3 - 6 } & 0.511 & 18.123 & 0.000 & & Tolerancia \\
\hline Constante & 0.103 & 27.754 & 0.000 & 0.542 & 2.211 \\
\hline Comunicación escrita & 0.107 & 28.872 & 0.000 & 0.391 & 2.558 \\
\hline Inglés & 0.321 & 63.627 & 0.000 & 0.264 & 3.782 \\
\hline Razonamiento cuantitativo & 84.300 & 0.000 & 0.257 & 3.893 \\
\hline Competencias ciudadanas & 0.444 & & & \\
\hline
\end{tabular}


Con el fin de detectar la linealidad del modelo y de la igualdad de varianzas, la Figura 1 muestra la distribución de la regresión del residuo tipificado y de la dispersión encontradas. Se verificó el cumplimiento de las condiciones exigidas para la regresión múltiple, encontrando que el modelo es adecuado en la explicación de las relaciones entre variables.
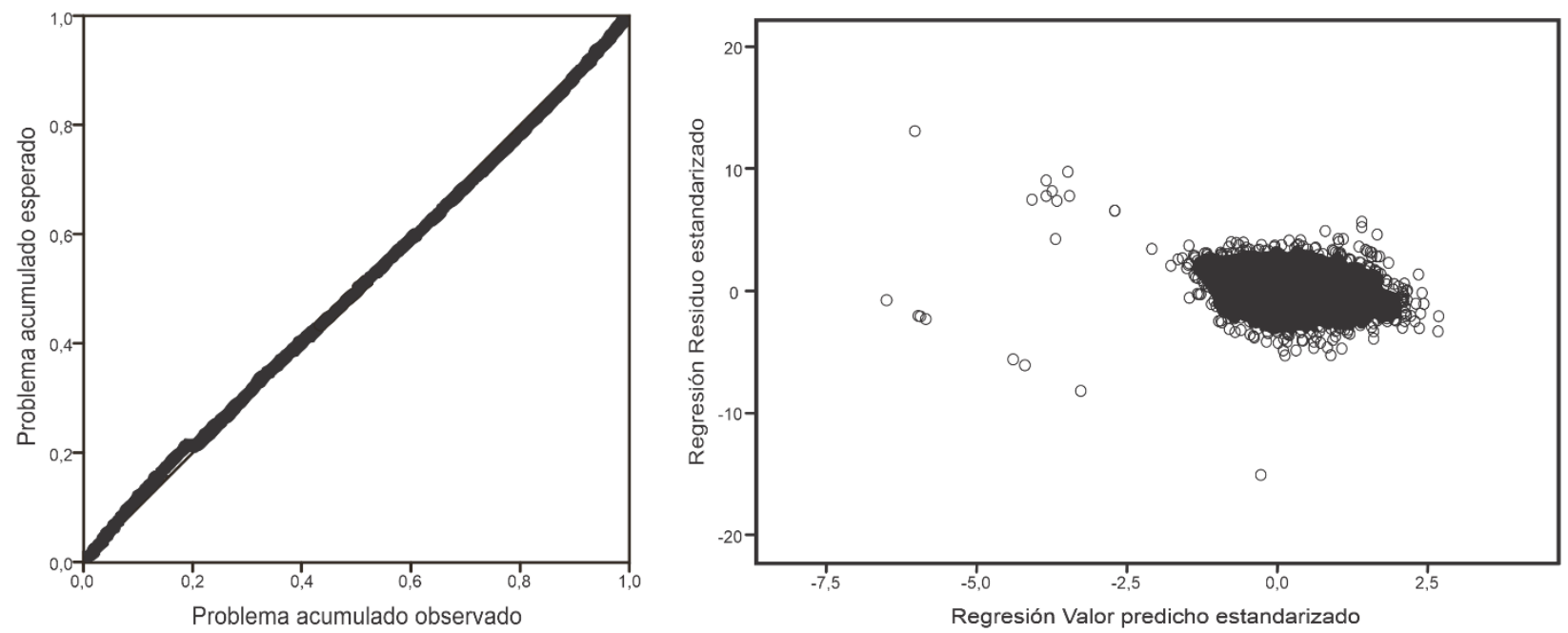

Fig.1: Prueba detección linealidad del modelo y de homocedasticidad.

\section{Comportamiento en Saber Pro para la Licenciatura en Etnoeducación}

Al efectuar la regresión múltiple para este programa se encuentran como estadísticos descriptivos los reportados en la Tabla 8. En la Tabla 8 todos los casos, los datos utilizados correspondieron a 405 registros. Para valorar las condiciones de fiabilidad y validez del modelo de regresión múltiple entre lectura crítica y los resultados de las cuatro pruebas, las Tablas 9 y 10 muestran los resultados encontrados. Con el fin de detectar la linealidad del modelo y de la igualdad de varianzas, la Figura 2 muestra la distribución de la regresión del residuo tipificado y de la dispersión encontradas.

Tabla 8: Estadísticos descriptivos de las competencias genéricas Saber Pro en la carrera de Licenciatura en Etnoeducación presentados en el año 2015

\begin{tabular}{|l|l|l|}
\hline Variable & Media & Desviación estándar \\
\hline Puntaje en lectura crítica & 9.707 & 1.5302 \\
\hline Puntaje en comunicación escrita & 9.490 & 1.7530 \\
\hline Puntaje en inglés & 9.771 & 1.9000 \\
\hline Puntaje en razonamiento cuantitativo & 9.118 & 1.4870 \\
\hline Puntaje en competencias ciudadanas & 9.401 & 1.5249 \\
\hline
\end{tabular}

Tabla 9: Estadísticos resumen del modelo de regresión lineal para lectura crítica

\begin{tabular}{|c|c|c|c|c|c|c|}
\hline \multirow{4}{*}{$\begin{array}{l}\text { Pruebas } \\
\text { Regresión } \\
\text { múltiple }\end{array}$} & \multicolumn{6}{|c|}{ Valores estadísticos } \\
\hline & \multirow{2}{*}{$\mathrm{R}_{\text {ajustado }}$} & \multirow[t]{2}{*}{ Error estándar } & \multicolumn{3}{|c|}{ Estadísticos de cambio } & \multirow[t]{2}{*}{ Durbin-Watson } \\
\hline & & & $\mathrm{R}_{\text {ajustado }}$ & $\mathrm{F}$ & Sig. $F$ & \\
\hline & 0.799 & 0.6853 & 0.011 & 21.589 & 0.000 & 2.067 \\
\hline \multirow[t]{4}{*}{ ANOVA } & Estimados & $\begin{array}{l}\text { Suma } \\
\text { cuadrados }\end{array}$ & gl & $\begin{array}{l}\text { Media } \\
\text { cuadrática }\end{array}$ & $\mathrm{F}$ & Sig. \\
\hline & Regresión & 757.655 & 3 & 252.552 & 537.66 & 0.000 \\
\hline & Residuo & 188.322 & 401 & 0.470 & & \\
\hline & Total & 945.978 & 404 & & & \\
\hline
\end{tabular}

Tabla 10: Coeficientes para el modelo de regresión múltiple de lectura crítica en la Licenciatura en Etnoeducación

\begin{tabular}{|l|l|l|l|l|l|}
\hline Variable & $\begin{array}{l}\text { Coeficientes } \\
\text { estandarizados }\end{array}$ & $t$ & Sig. & \multicolumn{2}{|l|}{ Estadísticas colinealidad } \\
\cline { 3 - 6 } & 0.766 & 3.381 & 0.001 & & Volerancia \\
\hline Constante & 0.550 & 13.826 & 0.000 & 0.316 & 3.62 \\
\hline Competencias ciudadanas & 0.276 & 7.040 & 0.000 & 0.342 & 2.921 \\
\hline Razonamiento cuantitativo & 0.133 & 4.646 & 0.000 & 0.465 & 2.151 \\
\hline Comunicación escrita & & & & \\
\hline
\end{tabular}



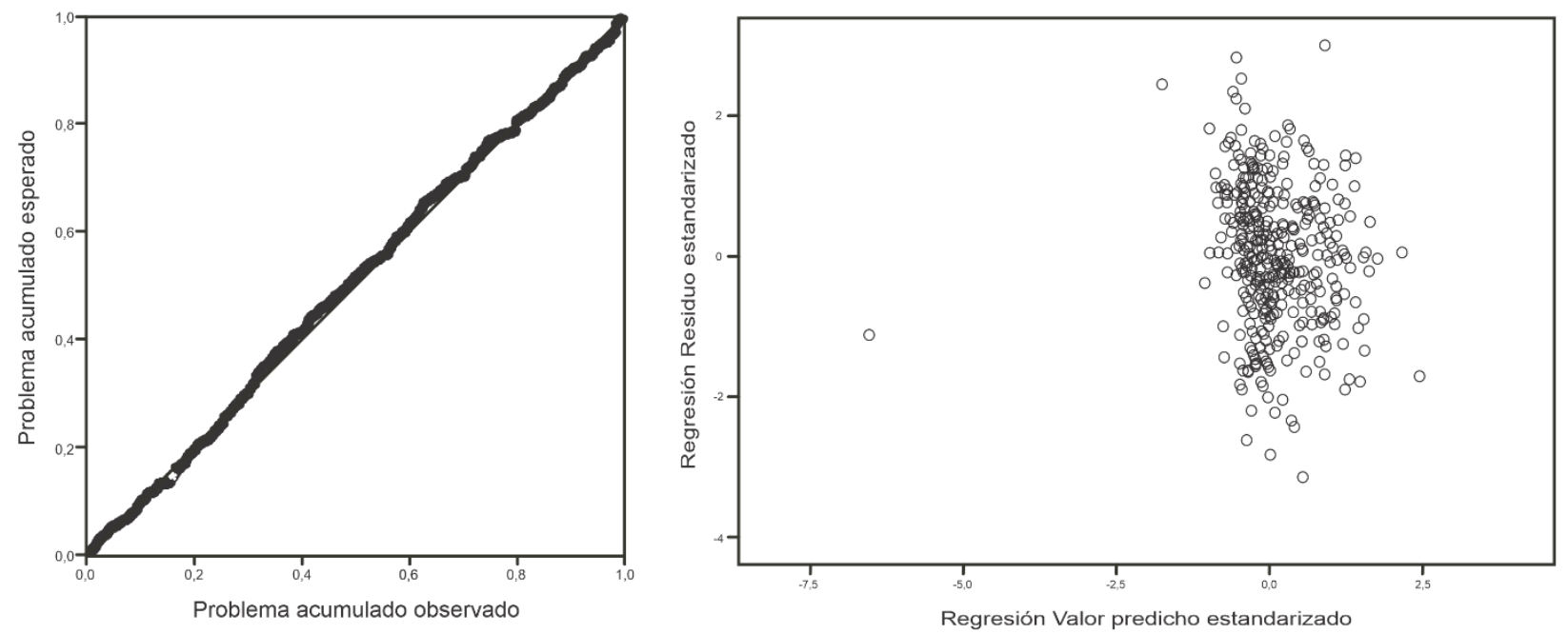

Fig. 2: Prueba detección linealidad del modelo y de homocedasticidad.

\section{DISCUSIÓN}

Los cuatro modelos econométricos encontrados se caracterizan por incluir variables explicativas significativas al $95 \%$ para el rendimiento académico obtenidos por los estudiantes de dos programas que a nivel nacional presentaron las pruebas de competencias genéricas Saber Pro del año 2015. En todos los casos se encontraron comportamientos diferenciales entre los dos programas, índice de que el rendimiento académico en estas pruebas estandarizadas depende de su naturaleza. Se empleó el modelo de regresión múltiple por ser adecuado para este tipo de estudios y el empleo de otras técnicas más complejas no aportan mucho a su mejoramiento, aunque es pertinente la observancia de las restricciones que tiene durante su aplicación (Kobrinet al., 2011).

En este estudio, se entiende como rendimiento académico a la medición cuantitativa de las respuestas de un individuo para demostrar su aprendizaje en una temática definida por una evaluación estandarizada (Zahed et.al., 2016). Así, se considera como variable dependiente el rendimiento total de los estudiantes en las pruebas Saber Pro y como variables explicativas: género del examinado, estado civil, carácter académico de la IES, sector al que pertenece la IES, si estuvo becado, usó crédito para pagar los estudios, si los padres le pagaron la matrícula, el estudiante pagó sus estudios, si el estudiante es cabeza de familia, el número de personas a cargo, el nivel educativo del padre, de la madre, ocupación laboral del padre, de la madre, el estrato de su vivienda, si trabaja y la metodología de estudio en la determinación del primer modelo global. En los dos casos inicialmente se definió el modelo ANOVA univariado encontrando los potenciales factores que determinarían un modelo ANOVA multivariado potencialmente explicativo. El otro interés del estudio correspondió buscar un modelo global explicativo entre el puntaje de la prueba Saber Pro de lectura crítica con los de las otras pruebas Saber Pro como comunicación escrita, inglés, razonamiento cuantitativo y competencias ciudadanas. Ahora, se discuten los resultados encontrados para los dos programas teniendo en cuenta cada uno de los modelos econométricos encontrados.

\section{Modelo econométrico global del rendimiento académico y algunas variables socioeconómicas}

Se modeló el rendimiento académico estableciendo la inferencia en el comportamiento de los resultados encontrados, proponiéndose inicialmente determinar esas relaciones con los 16 factores socioeconómicos. Dada la complejidad de los modelos de ANOVA univariados multifactoriales, primero se hizo una regresión lineal de cada factor con el rendimiento académico para encontrar cuáles de esas relaciones son significativamente explicativas considerando como criterio el valor del estadístico $\mathrm{R}^{2}$ ajustado esperando explicaciones de varianza superiores al 50\%. En esta forma, se encontró que para Administración de Empresas el modelo no mostró los mejores resultados ya que los $\mathrm{R}^{2}$ ajustados entre el rendimiento académico y cada uno de los factores no pudo explicar más allá del dos por ciento de la varianza; sin embargo, se probó el modelo ANOVA multifactorial con los factores que tuvieron ese valor. Tampoco fue exitoso ya que el modelo global subió hasta el 4,6\% de la varianza indicando que los factores considerados no explican con suficiencia el rendimiento de los estudiantes de este programa en las pruebas Saber Pro. En cambio, para la Licenciatura en Etnoeducación la situación fue diferente ya que el modelo ANOVA multifactorial encontró que cuatro factores que inicialmente explicaban cerca del $10 \%$ de la variabilidad en sus interacciones alcanzaron el $51,6 \%$ permitiendo comprender que en ese programa el sector de la IES, el nivel educativo alcanzado por el padre y la madre, la ocupación de la madre, el estrato y la modalidad de estudio se relacionan con el rendimiento académico alcanzado en la prueba Saber Pro. 


\section{Modelo econométrico para la relación del rendimiento en lectura crítica con los puntajes}

Las pruebas genéricas Saber Pro comprenden evaluaciones independientes del desempeño en lectura crítica, comunicación escrita, inglés, razonamiento cuantitativo y en competencias comunicativas. Se realizó la regresión múltiple con entrada de variables para definir el mejor modelo que se ajustara, correspondiendo a cuatro propuestas. En la sección de resultados se documentaron los valores de los estadísticos que permitieron modelar el rendimiento de lectura crítica en función de los puntajes de las otras cuatro variables.

Los dos modelos cumplieron con las cinco condiciones definidas para este tipo de regresiones múltiples mostrando relaciones significativas entre la variable dependiente rendimiento en lectura crítica y las otras variables explicativas relacionadas con el puntaje obtenido individualmente en cada una de las pruebas de competencias genéricas. Ahora bien y como con el modelo ANOVA multivariado, también son específicos del programa. Al comparar los estadísticos descriptivos de las puntuaciones en cada prueba se encuentran los valores relativamente más altos en los estudiantes de Administración que en los de la Licenciatura en todos los casos, aunque mantienen dispersiones semejantes en cada una de las pruebas.

Los valores del $\mathrm{R}^{2}$ ajustado en los dos modelos son altos y explican entre el 80 y el $84 \%$ de la varianza, con estadísticos de no colinealidad significativos (tablas 7 y 10) y gráficos de dispersión de residuos lineales y con distribución aleatoria (figuras 1 y 2), así es posible escribir la ecuación del modelo global para cada uno de los programas así:

$$
\begin{aligned}
& \text { LecCrit }_{\text {ADMON }}=0.511+0.109 \text { ComEsc }+0.122 \mathrm{Ing}+0.327 \text { RazCuan }+0.444 \text { CompCiud }+ \text { Error } \\
& \text { LecCrit }_{\text {LIEETN }}=0.766+0.548 \text { CompCiud }+0.268 \text { RazCuan }+0.152 \text { ComEsc }+ \text { Error }
\end{aligned}
$$

Comparando las ecuaciones (1) y (2) se encuentra que para Administración los puntajes de las competencias explicativas son positivas siendo las más importantes competencias ciudadanas y razonamiento cuantitativo, mientras que en la Licenciatura no es significativo el resultado en inglés quedando la relación explicada por los puntajes de tres competencias genéricas siendo también importantes los aportes de competencias comunicativas y de razonamiento cuantitativo. Algo para tener en cuenta en el desempeño diferencial de los estudiantes según el programa académico cursado.

\section{CONCLUSIONES}

De acuerdo al trabajo presentado y a los resultados obtenidos, se pueden plantear las siguientes conclusiones principales:

En la determinación de modelos econométricos para la explicación del rendimiento académico en las pruebas Saber Pro de 2015 en relación con 16 factores y conforme a los resultados encontrados para los programas de Administración de Empresas y de la Licenciatura en Etnoeducación, se estableció que los modelos ANOVA probados pudieron explicarlas para el último programa en función del sector de las IES, educación del padre, educación de la madre, ocupación de la madre, el estrato donde vive el estudiante y la modalidad de estudio y de las interacciones de dos de esas variables indicando que existen condiciones específicas de los programas relacionadas con las características que tienen los estudiantes.

Este estudio exploratorio sugiere que para buscar modelos explicativos más exitosos del rendimiento académico en pruebas estandarizadas en función de factores institucionales, socioeconómicos y familiares es necesario recurrir a otras técnicas como lo puede ser la regresión geográficamente ponderada que considera la ubicación espacial del examinado y permitiría la explicación de la región en esta variable dependiente.

En el modelo econométrico entre el rendimiento en lectura crítica y los puntajes de las pruebas Saber Pro comunicación escrita, inglés, razonamiento cuantitativo y competencias ciudadanas hay diferencias en el desempeño de los estudiantes por programa indicando nuevamente que las características y condiciones requeridas por los mismos hacen diferencial las relaciones que explicarían porque en Administración el inglés tiene influencia mientras que en Etnoeducación no.

\section{AGRADECIMIENTOS}

Proyecto PG-02-2017: Eficacia de programas a distancia, virtuales y presenciales en el aprendizaje de estudiantes de grado para el mejoramiento de la calidad, financiado por la Universidad Nacional Abierta y a Distancia - UNAD (Colombia) y la Fundación Universitaria Católica del Norte - FUCN (Colombia). 


\section{NOTACIÓN}

Ren $=$ Rendimiento en las pruebas Saber Pro

SECTOR $=$ Sector al que pertenece la IES

ESTRATO $=$ Estrato donde vive el estudiante

EDUCPADRE $=$ Nivel educativo del padre

EDUCMADRE $=$ Nivel educativo de la madre

OCUPMADRE = Ocupación de la madre

\author{
MODET $=$ Modalidad o metodología de estudio \\ LecCrit $=$ Puntaje en lectura crítica \\ ComEsc $=$ Puntaje en comunicación escrita \\ Ing = Puntaje en inglés \\ RazCuan = Puntaje en razonamiento cuantitativo \\ CompCiud $=$ Puntaje en competencias ciudadanas
}

\section{REFERENCIAS}

Adeyemi, A. y Adeyemi, S., Personal factors as predictors of students academic achievement in colleges of education in South Western Nigeria, https://doi.org/10.5897/ERR2014.1708, Educational Research and Reviews, 9(4), 97-109 (2014).

Ahumada, V., Gamboa, M. y Guerrero, J., Calidad de la educación superior en Colombia: Eficacia de algunos programas académicos presenciales y a distancia en las pruebas Saber Pro, 1-257, Sello Editorial Universidad Nacional Abierta y a Distancia, Bogotá, Colombia (2019).

Betancourt, F y Frías, L., Competencias argumentativas de los estudiantes de derecho en el marco de las pruebas Saber-Pro, http://dx.doi.org/10.22518/16578953.289, Civilizar, 15(28), 213-228 (2015).

Bustamante, M.A., Lapo, M., Oyarzún, C., y Campos, R.M., Análisis de la percepción del docente en tres universidades chilenas tras la implementación del currículum basado en competencias, http://dx.doi.org/10.4067/S071850062017000400009, Formación Universitaria, 10(4), 97-110 (2017).

Damary, R., Markova, T. y Pryadlina, N., Key Challenges of On-line Education in Multi-cultural Context. https://doi.org/10.1016/j.sbspro.2017.02.034, Procedia - Social and Behavioral Sciences, 237, 83-89 (2017).

De Clercq, M., Roland, N., Brunelle, M., Galand, B. y Frenay, M. The Delicate Balance to Adjustment: A Qualitative Approach of Student's Transition to the First Year at University, http://doi.org/10.5334/pb.409, Psychologica Belgica, 58(1), 67-90 (2018)

De La Hoz, E., Guillen, S. y Fontalvo, T., Análisis de la acreditación de calidad en programas de ingeniería industrial y los resultados en las pruebas nacionales estandarizadas, https://dx.doi.org/10.4067/S0718-50062020000100127, Formación universitaria, 13(1), 127-134 (2020).

Edel, N. R., El rendimiento académico: concepto, investigación y desarrollo, REICE Revista Iberoamericana sobre Calidad, Eficacia y Cambio en Educación. 1(2), 1-16 (2003).

European Commission., Teachers and school leaders in schools as learning organisations Guiding principles for policy development in school education. $2^{a}$ Ed., 1-68, Brussels, European Commission (2018).

Grifoll, J., Huertas, E., Prades, A., Rodríguez, S., Rubin, Y., Mulder, F. y Ossiannilsson E., Quality Assurance of Elearning, European Association for Quality Assurance in Higher Education. Helsinki, Finland (2010).

Gómez, V., Tolozano, M. y Delgado, N., The Institutional Accreditation of Quality in Technical and Technological Institutes of Higher Education in Ecuador from the Perspective of an Accredited Institute, http://dx.doi.org/10.4067/S0718-50062017000600007, Formación Universitaria, 10(6), 59-66 (2017).

Gutiérrez, D., Ortega, M., Henao, C., Torres, C. y Sánchez, J., Modelo para la construcción colectiva de conocimiento para la ruralidad, https://doi.org/10.22490/issn.2145-6453, Revista de Investigación Agraria y Ambiental RIAA, 10(2), 65-73 (2019).

Instituto Colombiano para la Evaluación de la Educación, ICFES., Guía de orientación Saber Pro 2019, 1-46, Bogotá, Colombia (2019).

Instituto Colombiano para la Evaluación de la Educación, ICFES., Guía introductoria al diseño centrado en evidencias. Bogotá: Instituto Colombiano para la Evaluación de la Educación. https://www.icfes.gov.co. (2018)

Kobrin, J. L., Sinharay, S., Haberman, S. J., y Chajewski, M. An investigation of the Fit of linear regression models to data from an SAT ${ }^{\circledR}$ validity study. New York: College Board Research, report 2011-3 (2011).

Londoño, E., Diseño instruccional para programas por competencias; En: El diseño instruccional: reflexiones y perspectivas en la Católica del Norte Fundación Universitaria. 59-78, Fundación Universitaria Católica del Norte, Medellín, Colombia (2013).

Markova, T., Glazkova, I. y Zaborova, E., Quality Issues of Online Distance Learning, https://doi.org/10.1016/j.sbspro.2017.02.043, Procedia - Social and Behavioral Sciences, 237, 685-691 (2017).

Martínez, C. P., y González, M. N., Las competencias transversales en la universidad: propiedades psicométricas de un cuestionario, https://doi.org/10.5944/educxx1.20194, Educación XXI, 21(1), 231-262 (2018).

Middlehurst, R., Changing Internal Governance: Are Leadership Roles and Management Structures in United Kingdom Universities Fit for the Future? https://doi.org/10.1111/hequ.12018, Higher Education Quarterly, 67(3), 275-294 (2013). 
Ministerio de Educación Nacional, MEN, Decreto 1330 de 2019 Por el cual se sustituye el Capítulo 2 y se suprime el Capítulo 7 del Título 3 de la Parte 5 del Libro 2 del Decreto 1075 de 2015 -Único Reglamentario del Sector Educación, Bogotá, Colombia (2019).

Ministerio de Educación Nacional, MEN., Competencias genéricas en Educación Superior, Boletín Informativo Educación Superior, 13, 1-20 (2009a).

Ministerio de Educación Nacional, MEN., Decreto 3963 por el cual se reglamenta el Examen de Estado de Calidad de la Educación Superior, 1-4, Bogotá, Colombia (2009b).

Olivos, P., Santos, A., Martín, S., Cañas, M., Gómez-Lázaro, E., y Maya, Y., The relationship between learning styles and motivation to transfer of learning in a vocational training programme, https://doi.org/10.1016/j.sumpsi.2016.02.001, Suma Psicológica, 23traductor(1), 25-32 (2016).

Pham, L., Williamson, S., y Berry, R., Student perceptions of e-learning service quality, e-satisfaction, and e-loyalty, https://doi.org/10.4018/IJEIS.2018070102, International Journal of Enterprise Information Systems, 14(3), 19-40 (2018).

Rausaria, R.R. y Lele, N.A., Self-Assessment of Distance Education Institutions: Identification of Parameters for Programme Assessment, Indian Journal of Open Learning, 11(1), 147-157 (2002).

Simonson, M. Smaldino, S. Albright, M. y Zvacek, S, Teaching and learning at a distance. Foundations of distance education, 5a. Ed., 1-374, MA: Pearson, Boston, USA (2012).

Soria, K. y Zúñiga, S., Aspectos determinantes del éxito académico de estudiantes universitarios, http://dx.doi.org/10.4067/S0718-50062014000500006, Formación universitaria 7(5), 41-50 (2014).

United Nations Educational, Scientific and Cultural Organization, UNESCO. World Conference on Higher Education: Vision and Action, 1-16, Paris, France (1998).

Zahed, Z., Rezaee, R., Yazdani, Z., Bagheri, S. y Nabeiei, P., The influence of parenting style on academic achievement and career path. Journal of advances in medical education \& professionalism, 4(3), 130-134 (2016).

Zhao, Y., Lei, J., y Yan, B, What makes the difference? A practical analysis of research on the effectiveness of distance education, https://doi.org/10.1111/j.1467-9620.2005.00544.x, Teachers College Record, 107(8), 1836-1884 (2005). 